\title{
Impact of "natural" cesarean delivery on peripartum blood loss: A randomized controlled trial
}

\author{
Noah Zafran ${ }^{1}$, Gali Garmi ${ }^{2}$, Suzan Abdelgani ${ }^{1}$, Shiri Inbar ${ }^{1}$, Shabtai Romano ${ }^{1}$, and Raed \\ Salim ${ }^{1}$ \\ ${ }^{1}$ Emek Medical Center \\ ${ }^{2}$ Haemek Center
}

August 3, 2020

\begin{abstract}
Objective: To examine the impact of "natural" cesarean deliveries (NCD) on peripartum maternal blood loss. Design: Randomized controlled trial. Setting: Single academic hospital in Afula, Israel, between August 2016 and February 2019. Population or Sample: Term singleton gestations scheduled for a planned CD under spinal anesthesia. Methods: Women were randomized at a ratio of 1:1 to NCD (study group) or traditional CD (control group). Women in the study group watched fetal extraction, had early skin to skin contact, and breastfed until the end of surgery. Neonates in the control group were presented to the mother without direct contact. Blood samples were drawn from all women to determine oxytocin levels using an ELISA kit. Main Outcome Measures: Postpartum hemoglobin ( $\mathrm{Hb}$ ) levels. Results: 214 women randomized, 23 were excluded. There were no significant differences in demographic and obstetric variables between the groups. Postpartum Hb levels were $10.1 \pm 1.1$ and $10.3 \pm 1.3 \mathrm{~g} / \mathrm{dL}$ in the study and control groups, respectively $(\mathrm{P}=.19)$. There were no significant differences in estimated blood loss, and rates of $\mathrm{PPH}$ and blood transfusion. Maternal pain scores, satisfaction, and exclusive breastfeeding rate at discharge, were similar. Maternal oxytocin blood levels were $389.5 \pm 183.7$ and $408.5 \pm 233.6 \mathrm{pg} / \mathrm{mL}$ in the study and control groups, respectively $(\mathrm{P}=.96)$. Rate of composite neonatal morbidity that comprised neonatal hypothermia, hypoglycemia, jaundice, and neonatal intensive care unit admission was higher in the study group. Conclusions: NCD does not affect maternal blood loss. Maternal oxytocin blood levels in NCD and traditional CD are similar.
\end{abstract}

\section{INTRODUCTION}

Early skin to skin contact (ESTSC) after vaginal delivery increases milk production and lactation and may improve maternal and neonatal outcomes. ${ }^{1-4}$ Studies published as early as the 1970s showed a change in maternal and neonatal behavior after ESTSC. ${ }^{1}$ Mothers practicing ESTSC and suckling, breastfed their infants longer than did routine-care mothers. ${ }^{2}$ A Cochrane meta-analysis concluded that ESTSC promotes breastfeeding, though other conclusions were challenging due to methodological quality and small sample size of the included trials. ${ }^{3}$

Breastfeeding and ESTSC post postpartum, increase endogenous oxytocin release into circulating blood, ${ }^{4-9}$ thus minimizing the risk of postpartum hemorrhage (PPH). ${ }^{9}$ A retrospective study found that women who did not have ESTSC were almost twice as likely to have PPH compared to women who both had ESTSC and began breastfeeding within 30 minutes of birth. ${ }^{10}$

Although the delivery goal for most women is to experience a vaginal birth, a cesarean delivery (CD) is sometimes mandatory due to medical maternal or fetal indications. Recent attention has focused on the description and putting into practice of techniques in the operating ward to incorporate ESTSC and breastfeeding. The first approach of modifying the CD was described in 2008 by Smith et al. and was termednatural $C D$ (NCD). ${ }^{11}$ One of the main aspects was to mimic the situation of vaginal birth and 
to give parents a better birthing experience (family-centered CD). Nevertheless, there is a lack of adequate methodological studies on the impact of CD incorporating ESTSC and breastfeeding, i.e., NCD, and maternal outcomes. ${ }^{11}$

The present trial aims to examine the effect of NCD on peripartum blood loss compared to traditional CD and to examine the impact on women's satisfaction, exclusive breastfeeding, and other maternal and neonatal outcomes.

\section{PATIENTS AND METHODS}

This randomized controlled trial was conducted in the labor and delivery ward of a single university teaching hospital in Afula, Israel. The clinical trial was conducted between August 2016 and February 2018, while the laboratory component was completed in February 2019.

Pregnant women scheduled for planned CD at our institution were given the option to participate in the trial. Eligible women were randomly assigned in a 1:1 ratio to NCD (study group) or traditional CD (control group). The inclusion criteria of both groups included maternal or fetal indication for CD, planned CD under spinal anesthesia at term, maternal age between 18 and 45 years, viable fetus, and confirmed dating by last menstrual period and first- or early second-trimester ultrasound. Women with unplanned CD, had multifetal gestation, major fetal malformations, fetal conditions requiring immediate neonatologist evaluation, estimated fetal weight below the 5 th percentile, noncontrolled diabetes mellitus, or severe preeclampsia were excluded, as were women who were human immunodeficiency virus carriers or who were unable to give informed consent. Women were excluded from the analysis after recruitment if they had failed spinal anesthesia, thus requiring general anesthesia, or if their newborns needed immediate medical care after delivery.

In the study group only, the intravenous line was inserted in the nondominant hand. In the operating room, the blood pressure cuff was placed on the nondominant side or on the leg. The pulse oximeter was placed on the toe. Women's arms were not tied down, and at least one arm was free from clothing. The electrocardiogram stickers were placed on the mother's back, leaving the chest free. The drape separating the surgical field was placed closer to the abdomen in a way that allowed smooth lowering down and created a relatively large free chest area. At head delivery, the surgical drape was lowered in women electing to watch the slow "extraction" of the baby out. The scrubbed midwife received the neonate from the surgeon, dried and directly placed the neonate on the naked skin of the mother's breast to initiate ESTSC and encourage suckling. The neonate was covered with a warm blanket. At this stage, the surgical drapes were lifted up again before removing the placenta. Usual neonatal care, such as assigning an Apgar score and placing a name tag, was done during ESTSC. Weighing the neonate was postponed until ESTSC was terminated. The midwife remained alongside the mother constantly as long as the neonate was on the mother's chest. ESTSC was terminated on maternal request, at any compromise in maternal or neonatal well-being mandating medical care, or at the end of the surgical procedure.

Women in the control group, i.e., traditional CD, were not given the option to watch extraction and did not breastfeed during surgery. After the midwife received the neonate and assessed the need for medical care, she dried, weighed, name tagged, and covered the neonate. Following that, the neonate was held adjacent to its mother, or was given to the escort to be held for a few minutes and then was transferred to the nursery unit.

In both groups, complete blood count (CBC) was taken on admission as part of surgical preparation. Antibiotic prophylaxis before skin incision was given to all participants prior to surgical incision. Women in both groups were allowed an escort of their choice in the surgery suite after confirmation of adequate spinal analgesia. Delayed cord clamping for nearly 60 seconds was made similar. Uterotonic medications to prevent $\mathrm{PPH}$ were given according to the department protocol; following the delivery of the neonate, 5 units of oxytocin was given in a slow intravenous push followed by 15 units in a 1000-mL lactated Ringer's solution as the standard regimen. Higher doses of oxytocin or the use of other uterotonic medications were considered if needed to treat uterine atony. Other surgical techniques were identical between the 2 groups. Room temperature at the operating suit was set at $25^{\circ} \mathrm{C}$. Perioperative analgesics or sedatives were given according 
to the anesthesiologist's discretion.

Maternal oxytocin levels were examined in both groups to attain another reliable evidence of the impact of ESTSC and lactation on oxytocin release. For that purpose, blood samples were obtained from all women during fascia closure and were subsequently analyzed in a designative laboratory for determining oxytocin levels among the groups using an ELISA kit (IBL International GmbH: RE52331; Flughafenstrasse 52a, D22335 Hamburg, Germany). Blood samples were drawn into chilled serum or EDTA (1 mg/mL blood) tubes containing aprotinin $(500 \mathrm{KIU} / \mathrm{mL}$ of blood) and were centrifuged within 10 minutes at $1600 \mathrm{x}$ g for 15 minutes at $4^{\circ} \mathrm{C}$. The serum of all participants was stored in a plastic tube at $-70^{\circ} \mathrm{C}$ until kit application after completion of the clinical trial. Oxytocin extraction from an equal volume $(500 \mu \mathrm{l})$ of serum spiked with 100 pg of oxytocin across all samples was carried out with a standard protocol using the C18 Sep-Pak column, according to the manufacturer's instructions. An oxytocin standard curve was provided in the ELISA kit with a limit of detection of $15 \mathrm{pg} / \mathrm{mL}$.

Postoperative pain relief medication during hospitalization was given according to a department protocol. Postoperative pain score was measured according to visual analog scale (VAS) from 0 to $10 \mathrm{~cm}$, whereno pain scored 0 and worst pain scored 10.

The morning after surgery, a routine CBC was drawn. Since this routine CBC may not reflect post-surgical nadir hemoglobin $(\mathrm{Hb})$ level, additional $\mathrm{CBC}$ was drawn 2 to 3 days after delivery, as part of the study protocol. In cases of blood transfusion, $\mathrm{Hb}$ nadir recorded before transfusion, was included as a substitute in the analysis. Other blood tests were drawn according to clinical judgment. Prior to discharge, women were asked to complete a self-reporting satisfaction questionnaire related to their childbirth experience. A satisfaction questionnaire that was previously validated for use in clinical trials was used. ${ }^{12}$ The questionnaire contains 38 questions, and each answer scores on a scale of 1 to $6(1=$ least satisfied and $6=$ most satisfied ). An average score was calculated rather than the total score, since a number of women did not answer all questions because they assumed that some were not relevant.

Neonates in both groups were assessed at the nursery on admission, including measurement of temperature and glucose level as indicated.

\section{Outcome Measures}

The primary outcome examined was the level of postpartum $\mathrm{Hb}$ at 2 to 3 days after delivery. Secondary outcomes included the need for additional use of perioperative uterotonics, the need for blood transfusion, maternal pain scores, the need for additional analgesia or sedatives during and in the immediate postoperative period, maternal infections until discharge, exclusive breastfeeding at discharge, maternal satisfaction, and maternal oxytocin levels. Neonatal outcomes included Apgar scores and a composite morbidity that comprised neonatal hypothermia, hypoglycemia, jaundice, and neonatal intensive care unit (NICU) admission.

\section{Sample size}

Power analysis was based on $\mathrm{Hb}$ level after $\mathrm{CD}$. We examined $\mathrm{Hb}$ levels of 150 women who underwent planned $\mathrm{CD}$ during the year before study initiation. The average $\mathrm{Hb}$ level found was $10.4 \pm 1.3 \mathrm{~g} / \mathrm{dL}$. In order to detect a $0.5 \mathrm{~g} / \mathrm{dL}$ difference in favor of women undergoing NCD, 214 women in both groups were needed with a level of significance of $95 \%(\alpha=.05)$ and a power of $80 \%(\beta=0.2)$. The analysis was performed according to the intention-to-treat principle.

\section{Randomization}

Before women enrollment by the investigators, a computer randomization sequence generation was used to produce the randomization. Eligible women who signed informed-consent forms were randomly assigned in a 1:1 ratio to one of the two groups. The randomization sequence was prepared by the primary investigator and was kept in a sealed envelope. The sequence was concealed until intervention was assigned. Women were allocated to randomization code numbers in chronological order. Due to the nature of the interven- 
tion, women and the dedicated staff were not blinded to group allocation. The data analysis individuals were unaware of the group assignments. Quality control of screening, handling of data, and verification of compliance to protocol was implemented by a local data monitoring committee.

\section{Statistical analysis}

A series of $\chi^{2}$ tests or Fisher exact tests (when the assumptions of the parametric $\chi^{2}$ test had not been met) and Student $t$ tests or nonparametric Mann-Whitney U tests (in the case where the underlying distribution was not normal) were conducted to analyze the differences between characteristics of the women in both groups. We computed the 2 -tailed $P$ values, where $P<.05$ was considered a statistically significant result. Statistical analyses were performed using the SAS software package version 9.4 (SAS Institute, Cary, NC).

Oxytocin levels were calculated using a 4-parameter logistic curve-fitting model (Excel, Microsoft), and results were compared using a 2-tailed Student $t$ test.

\section{RESULTS}

During the study period, 7240 deliveries took place; of those, 1286 were cesarean deliveries (17.8\%). Of all CDs, 625 were planned (48.6\%). Of all planned CDs, 214 eligible women gave consent and were randomized to either NCD $(n=108)$ or to traditional CD $(n=106)$. Of all randomized women, $23(10.7 \%)$ women were excluded from the final analysis: 7 ( 2 in the study and 5 in the control group) had general anesthesia due to failed spinal, 5 ( 2 in the study and 3 in the control group) underwent emergent CD within 24 hours before the scheduled CD, and 11 withdrew consent (2 and 9 in the study and control groups, respectively), leaving 102 women assigned to NCD and 89 to traditional CD (Figure 1). Among the study group, all had ESTSC. Forty-two $(41.2 \%)$ women succeeded to breastfeed during the entire period of surgery. Twenty-six (25.5\%) elected to watch extraction. None of the controls had ESTSC or breastfed.

Demographic and obstetric data of the study and control groups were comparable and are presented in Table 1. Postpartum Hb levels were $10.1 \pm 1.1$ and $10.3 \pm 1.3 \mathrm{~g} / \mathrm{dL}$ in the study and control groups, respectively $(P$ $=.19)$. Differences in $\mathrm{Hb}$ and hematocrit levels before and after the cesarean did not differ as well. Estimated blood loss $>1000 \mathrm{~mL}$ and use of uterotonic agents did not differ between the groups (Table 2). The length of maternal stay and rate of scar infection were comparable between the groups. Exclusive breastfeeding rate at discharge also did not differ between the groups $(P=.39$; Table 2$)$.

Pain scores were comparable between the groups as well as analgesia use during the operation and in the immediate postoperative period. The maternal satisfaction score was similar between the 2 groups $(5.4 \pm 0.74$ and $5.4 \pm 0.5$ in the study and control groups, respectively; $P=.68$; Table 3 ).

Subgroup analysis was performed among the NCD group, comparing only women who succeeded to breastfeed during the entire surgery to the controls. The primary outcome, i.e., postsurgical Hb levels, was comparable between the 2 groups $(P=.88)$. There was a higher rate of exclusive breastfeeding at discharge $(52.4 \%$ and $33.7 \%$ in the study and control groups, respectively; $P=.04$ ). Other outcomes examined did not differ significantly between the groups.

Neonatal outcomes are presented in Table 4. None differed between the groups; nevertheless, the incidence of composite morbidity that included neonatal hypothermia, hypoglycemia, jaundice, and NICU admission was higher among the NCD group compared to the controls $(P=.04)$.

Oxytocin levels were successfully determined in 182 out of 191 women (95.3\%) who were included for analysis of the primary outcome (97 and 85 in the study and control groups, respectively). There were no significant differences in demographic and obstetric variables or $\mathrm{Hb}$ levels before or after surgery between the groups. Maternal oxytocin blood levels were $389.5 \pm 183.7$ and $408.5 \pm 233.6 \mathrm{pg} / \mathrm{mL}$ in the study and control groups, respectively $(P=.96)$.

\section{DISCUSSION}

Main findings 
The results of this randomized trial involving women designated to have a planned CD at term demonstrate that NCD leads to a similar perioperative blood loss compared to traditional CD. Perioperative Hb-level decline, blood transfusion rate, and total perioperative uterotonics use were identical. Moreover, ESTSC and breastfeeding resulted in comparable maternal oxytocin levels, as measured via the ELISA kit, compared to traditional CD. Furthermore, maternal pain scores, need for additional analgesia or sedatives during the operation and in the immediate postoperative period, and maternal satisfaction were all comparable. There were no adverse maternal or perioperative effects related to NCD. In terms of neonatal outcome, there was no difference in any particular outcome examined; nevertheless, the incidence of composite morbidity that included hypothermia, hypoglycemia, jaundice, and NICU admission was higher among the study compared to the control group.

\section{Strengths and limitations}

The strengths of this study are its randomized nature and the objective measures examined. Measuring oxytocin levels is a substantial contribution that further strengthens the results. Though there is a paucity of literature regarding postpartum oxytocin norms, similar values have been reported earlier using an ELIZA kit as well, among pregnant women who did not have PPH. ${ }^{13}$

Limitations of this study are worth mentioning. The trial was not designed to detect significant differences in secondary outcomes. Furthermore, due to the nature of the intervention, blinding was not possible. Additionally, compared to multicenter studies, interventions examined in a distinct institution may be less generalizable. However, an advantage linked to a single-center trial is that the same surgical, perinatal, neonatal, and laboratory teams managed all the cases and applied the same peripartum management and oxytocin measurement technique.

\section{Interpretation}

Administration of synthetic oxytocin in the third stage of labor has been approved in reducing the rate of PPH. ${ }^{14}$ Synthetic oxytocin has a half-life of 1-2 minutes. ${ }^{15}$ In comparison, endogenous oxytocin is released in a pulsatile mode, has a half-life of $20-30$ minutes and surges in the third stage of labor. ${ }^{15}$ Therefore, endogenous oxytocin release is considered much more effective in maintaining uterine contractility compared with the synthetic oxytocin. ${ }^{9}$ Given that, it is reasonable to suggest that the reduction of the occurrence of PPH in cases of NCD as previously reported is related to both ESTSC and breastfeeding, which stimulate endogenous oxytocin release. ${ }^{4,5,9}$ Accordingly, the effect is supposed to be absent or diminished with the separation of the newborn from the mother immediately after birth. Separation also has been reported to create a state of distress that may block the release of oxytocin, and atony may result. ${ }^{9,10}$ A previous study found that women who did not practice ESTSC or did not breastfeed within 30 minutes of birth were almost twice as likely to have $\mathrm{PPH},{ }^{10}$ although the study had several limitations, including the retrospective nature that could lead to several errors. In addition, the association found may be related to reverse causation, i.e., absent ESTSC due to PPH, as the authors stated. Moreover, the collected data were not controlled, and the ESTSC technique was not standardized.

Lack of effect of ESTSC on blood loss, shown in the present study, was also described in a randomized trial among women delivered vaginally. ${ }^{16}$ The comparable oxytocin levels found in the current study support this observation.

There is little information on pain scores and the need for analgesia or sedatives during CD combined with ESTSC. The findings of the current study showed that pain scores and the use of additional analgesia or sedatives during surgery and the immediate postpartum hours were comparable. The results are consistent with the finding of Nolan et al. ${ }^{17}$ Others reported a decrease in the need for sedatives in women who elected to breastfeed during $\mathrm{CD}$; however, this study was not controlled, and the results were compared to historical control. ${ }^{18}$

Maternal satisfaction was comparable between the study and the control group. In contrast, Armbrust et al. showed in a randomized trial that ESTSC improved both maternal satisfaction and rate of breastfeeding. ${ }^{19}$ 
Nevertheless, the groups were not entirely similar, since women from the ESTSC group had a significantly higher level of education, a factor that may affect satisfaction. Additionally, Baethge et al. ${ }^{20}$ reported that influential conclusions could not be drawn from this study ${ }^{17}$ due to an additional number of errors in design and interpretation.

The impact of NCD on exclusive breastfeeding at discharge is conflicting. ${ }^{21}$ Prior studies have found an increase in exclusive breastfeeding rates for women undergoing a CD from $8 \%$ to $19 \%$ following initiation of ESTSC in the operating ward. ${ }^{22}$ Although exclusive breastfeeding was comparable between the groups in the current study, both groups had higher rates at discharge (nearly $34 \%$ to $43 \%$ ) compared to the cited article.

There is a paucity of literature regarding the impact of ESTSC on neonatal outcomes. ESTSC was described as a helpful tool in maintaining neonatal thermoregulation and blood glucose level after vaginal delivery, ${ }^{23}$ yet hypothermia after a cesarean can occur. ${ }^{24}$ Consistent with another report, ${ }^{25}$ the results of the current study did not show a difference in any particular neonatal outcome examined; nevertheless, a composite of neonatal morbidity that included hypothermia, hypoglycemia, jaundice, and NICU admissions was higher in cases of NCD compared to traditional CD. Similarly, one study reported an increase in the rate of unplanned NICU admission from $7 \%$ to $21 \%$ after the introduction of family-centered CD. ${ }^{21}$

Following the promising publication of NCD in 2008 by Smith et al., ${ }^{11}$ the editor commented that no outcomes or safety data are presented to justify widespread use of this technique and that the technique should be adequately studied with appropriate clinical trials. ${ }^{26}$ Additionally, Newman et al. reported that the term natural implies a process associated with fewer adverse outcomes than the traditional technique, although the practice changes suggested by Smith et al. do not reduce any significant adverse effects related to $\mathrm{CD} .{ }^{27}$ To date, only small-sample-size studies regarding safety have been published, ${ }^{28,29}$ with a lack of consistency and missing data that do not enable the drawing of conclusions.

NCD, according to the present trial, does not improve maternal health or surgical results. The procedure may be accompanied with higher expenses, mainly due to consuming the time of the nursing staff. Additionally, disappointment may be expressed by women and families when NCD is not available because of a shortage of nurse staffing and equipment. ${ }^{29}$

\section{Disclosure of interests}

All authors have no conflicts of interest.

\section{Contribution to authorship}

NZ, and RS contributed to concept and design of the study.

NZ, GG, SA, SI recruited participants, collected data and blood exams and prepared data for analysis.

NZ, GG, SI, SI, SR and RS reviewed data, revised statistical analysis and drafted the manuscript.

RS revised final version.

\section{Details of ethics approval}

The local institutional review board approved the study protocol (registration number 0137-15-EMC) in June 2016.

Clinical Trial Registration: clinicaltrials.gov Identifier: NCT02768142.

\section{Funding/support}

The described project was supported by The Foundation for Encouraging Excellence, Emek Medical Center, Afula, Israel. The funders had no role in the design or conduct of this study; collection, management, analysis, or interpretation of the data; preparation, review, or approval of the manuscript; or decision to submit this manuscript for publication.

\section{Acknowledgment}


The authors thank Dr. Amir Grau, Ph.D., Flow, and Mass Cytometry Center, Biomedical Core Facilities, Technion, Haifa, Israel, for performing and analyzing oxytocin levels using the ELISA kit. Additionally, the authors thank Dr. Julia Carmeli, MD, head of obstetric Anesthesiology unit, Emek Medical Center, Afula, Israel, for the productive cooperation and assistance with employing the study protocol in the operating theater.

\section{References}

1. De Chateau P, Wiberg B. Long-term effect on mother-infant behaviour of extra contact during the first hour postpartum. I. First observations at 36 hours. Acta Paediatr Scand. 1977;66:137-43.

2. De Château P, Wiberg B. Long-term effect on mother-infant behaviour of extra contact during the first hour post partum. III. Follow-up at one year. Scand J Soc Med. 1984;12:91-103.

3. Moore ER, Bergman N, Anderson GC, Medley N. Early skin-to-skin contact for mothers and their healthy newborn infants. Cochrane Database Syst Rev. 2016;11:CD003519.

4. Uvnäs Moberg K., Prime D.K. Oxytocin effects in mothers and infants during breastfeeding. Infant. 2013; 9: 201-6.

5. Christensson K, Nilsson BA, Stock S, Matthiesen AS, Uvnäs-Moberg K. Effect of nipple stimulation on uterine activity and on plasma levels of oxytocin in full term, healthy, pregnant women. Acta Obstet Gynecol Scand. 1989;68:205-10.

4. Drewett RF, Bowen-Jones A, Dogterom J. Oxytocin levels during breast-feeding in established lactation. Horm Behav. 1982;16:245-8.

7. Chua S, Arulkumaran S, Lim I, Selamat N, Ratnam SS. Influence of breastfeeding and nipple stimulation on postpartum uterine activity. Br J Obstet Gynaecol. 1994;101:804-5.

8. Abedi P, Jahanfar S, Namvar F, Lee J. Breastfeeding or nipple stimulation for reducing postpartum haemorrhage in the third stage of labour. Cochrane Database Syst Rev. 2016;1:CD010845.

9. Saxton A, Fahy K, Hastie C. Effects of skin-to-skin contact and breastfeeding at birth on the incidence of PPH: A physiologically based theory. Women Birth. 2014;27:250-3.

10. Saxton A, Fahy K, Rolfe M, Skinner V, Hastie C. Does skin-to-skin contact and breast feeding at birth affect the rate of primary postpartum haemorrhage: results of a cohort study. Midwifery. 2015;31:1110-7.

11. Smith J, Plaat F, Fisk NM. The natural caesarean: a woman-centred technique. BJOG. 2008;115:1037-42.

12. Lomas J, Dore S, Enkin M, Mitchell A. The Labor and Delivery Satisfaction Index: the development and evaluation of a soft outcome measure. Birth. 1987;14:125-9.

13. Jobst A, Krause D, Maiwald C, Härtl K, Myint AM, Kästner R, et al. Oxytocin course over pregnancy and postpartum period and the association with postpartum depressive symptoms. Arch Womens Ment Health.2016;19:571-9.

14. Practice Bulletin No. 183 summary: postpartum hemorrhage. Obstet Gynecol. 2017;130:923-5.

15. Ludwig M, Leng G. Dendritic peptide release and peptide-dependent beha-viours. Nat Rev Neurosci. 2006; $7: 126-36$.

16. Bullough CH, Msuku RS, Karonde L. Early suckling and postpartum haemorrhage: controlled trial in deliveries by traditional birth attendants. Lancet. 1989;2:522-5.

17. Nolan A, Lawrence C. A pilot study of a nursing intervention protocol to minimize maternal-infant separation after cesarean birth. J Obstet Gynecol Neonatal Nurs. 2009;38:430-42.

18. Sundin CS, Mazac LB. Implementing skin-to-skin care in the operating room after cesarean birth. $M C N$ Am J Matern Child Nurs. 2015;40:249-55. 
19. Armbrust R, Hinkson L, von Weizsäcker K, Henrich W. The Charité cesarean birth: a family orientated approach of cesarean section. J Matern Fetal Neonatal Med. 2016;29:163-8.

20. Baethge C, Blettner M, Friese K. Armbrust et al. 2015: randomization questionable. J Matern Fetal Neonatal Med 2016;29:3730-1.

21. ACOG Committee Opinion No. 766: approaches to limit intervention during labor and birth. Obstet Gynecol. 2019;133:e164-e73.

22. Brady K, Bulpitt D, Chiarelli C. An interprofessional quality improvement project to implement maternal/infant skin-to-skin contact during cesarean delivery. J Obstet Gynecol Neonatal Nurs. 2014;43:488-96.

23. Nimbalkar SM, Patel VK, Patel DV, Nimbalkar AS, Sethi A, Phatak A. Effect of early skin-to-skin contact following normal delivery on incidence of hypothermia in neonates more than $1800 \mathrm{~g}$ : randomized control trial. J Perinatol. 2014;34:364-8.

24. Horn EP, Bein B, Steinfath M, Ramaker K, Buchloh B, Hocker J. The incidence and prevention of hypothermia in newborn bonding after cesarean delivery: a randomized controlled trial. Anesth Analg. 2014;118:997-1002.

25. Billner-Garcia R, Spilker A, Goyal D. Skin to skin contact: newborn temperature stability in the operating room. MCN Am J Matern Child Nurs. 2018;43:158-63.

26. Editor's Commentary for: Smith J, Plaat F, Fisk NM. The natural caesarean: a woman-centred technique. BJOG. 2008;115:1037-42.

27. Newman L, Hancock H. How natural can major surgery really be? A critique of "the natural caesarean" technique. Birth. 2009;36:168-70.

28. Stevens J, Schmied V, Burns E, Dahlen H. Immediate or early skin-to-skin contact after a Caesarean section: a review of the literature. Matern Child Nutr. 2014;10:456-73.

29. Schorn MN, Moore E, Spetalnick BM, Morad A. Implementing family-centered cesarean birth. J Midwifery Womens Health. 2015;60:682-90.

Table 1: Demographic and obstetric variables of women according to trial group.

\begin{tabular}{llll}
\hline Variable & $\begin{array}{l}\text { Natural cesarean } \\
(\mathrm{N}=102)\end{array}$ & Control group (N=89) & P-value \\
\hline Maternal age, years & $33.0 \pm 5.2[33.0$, & $32.7 \pm 5.4[34.0$, & 0.99 \\
& $29.0-37.0]$ & $28.0-36.0]$ & \\
Ethnicity Arabs & $42(41.2) 60(58.8)$ & $47(52.8) 42(47.2)$ & 0.11 \\
Jews & & \\
Gravidity & $4.1 \pm 2.5[4.0,3.0-5.0]$ & $4.0 \pm 2.2[4.0,3.0-5.0]$ & 0.55 \\
Parity & $2.1 \pm 1.1[2.0,1.0-3.0]$ & $2.1 \pm 1.4[2.0,1.0-3.0]$ & 0.76 \\
Gestational age at & $38.5 \pm 0.6[38.3$, & $38.4 \pm 0.74[38.3$, & 0.54 \\
delivery, weeks & $38.1-38.6]$ & $38.1-39.0]$ & \\
Pregestational body & $25.7 \pm 5.1[24.6$, & $27.1 \pm 6.0[26.0$, & 0.12 \\
mass index $\left(\mathrm{kg} / \mathrm{m}^{2}\right)$ & $22.8-28.2]$ & $22.6-31.2]$ & \\
Indication for cesarean $_{\text {Previous uterine scar }}$ & $81(79.4) 15(14.7) 6(5.9)$ & $64(71.9) 13(14.6) 12$ & 0.60 \\
Fetal $^{1}$ Maternal & & $(13.5)$ & \\
Any diabetes & & & \\
Any hypertension $_{\text {Thrombophilia }}$ & $2(1.8)$ & $7(19.8)$ & 0.07 \\
& $7(6.9)$ & $5(5.6)$ & 0.08 \\
& &
\end{tabular}




\begin{tabular}{llll}
\hline Variable & $\begin{array}{l}\text { Natural cesarean } \\
(\mathrm{N}=102)\end{array}$ & Control group (N=89) & $P$-value \\
\hline $\begin{array}{l}\text { Mode of conception } \\
\text { Spontaneous Any fertility }\end{array}$ & $95(93.1) 7(6.9)$ & $84(94.4) 5(5.6)$ & 0.72 \\
treatment $^{3}$ & & \\
Hemoglobin prior & $11.4 \pm 1.1[11.4$, & $11.5 \pm 1.2[11.6$, & 0.63 \\
cesarean, g/dL & $10.7-12.0]$ & $10.9-12.2]$ & \\
Hematocrit prior & $34.0 \pm 2.9[33.8$, & $34.3 \pm 2.8[34.3$, & 0.44 \\
cesarean, \% & $32.1-35.8]$ & $32.6-36.1]$ & \\
Surgical length, & $43.4 \pm 12.5[41.8$, & $45.2 \pm 13.7[43.9$, & 0.36 \\
minutes & $34.2-49.3]$ & $35.0-51.4]$ & 0.40 \\
Tubal sterilization & $19(18.6)$ & $21(23.6)$ & \\
during cesarean & $12(11.8) 84(82.3) 6(5.9)$ & $12(13.5) 74(83.1) 3(3.4)$ & 0.69 \\
Skin closure Staples & & & \\
Intracuticular sutures & & & \\
Glue & & & \\
\hline
\end{tabular}

Data are mean \pm standard deviation [median, IQR] or $\mathrm{N}(\%)$ unless otherwise specified.

${ }^{1}$ Fetal indications: Breech presentation, suspect macrosomia, prior shoulder dystocia.

${ }^{2}$ Maternal indications: placenta previa or low lying placenta, maternal request.

${ }^{3}$ Fertility treatment: Clomiphene citrate, gonadotropin induction of ovulation and in vitro fertilization.

Table 2: Peripartum maternal outcomes according to trial group.

\begin{tabular}{|c|c|c|c|}
\hline Outcome & $\begin{array}{l}\text { Natural cesarean } \\
(\mathrm{N}=102)\end{array}$ & Control group $(\mathrm{N}=89)$ & $P$-value \\
\hline $\begin{array}{l}\text { Hemoglobin after } \\
\text { cesarean, g/dL }\end{array}$ & $\begin{array}{l}10.1 \pm 1.1[10.1 \\
9.5-10.9]\end{array}$ & $\begin{array}{l}10.3 \pm 1.3[10.2 \\
9.5-11.0]\end{array}$ & 0.19 \\
\hline $\begin{array}{l}\text { Hematocrit post } \\
\text { cesarean, } \%\end{array}$ & $\begin{array}{l}30.5 \pm 3.1[30.8 \\
28.6-32.6]\end{array}$ & $30.9 \pm 3.1[30.6,9-33.1]$ & 0.46 \\
\hline $\begin{array}{l}\text { Estimated blood loss } \\
>1000 \mathrm{~mL}\end{array}$ & $5(4.9)$ & $7(7.9)$ & 0.4 \\
\hline $\begin{array}{l}\text { Need for additional } \\
\text { uterotonics }\end{array}$ & $15(14.7)$ & $13(14.6)$ & 0.98 \\
\hline Blood transfusion & $1(1.0)$ & $0(0.0)$ & 0.99 \\
\hline Scar infection & $2(1.96)$ & $0(0.0)$ & 0.50 \\
\hline $\begin{array}{l}\text { Length of maternal } \\
\text { hospitalization, days }\end{array}$ & $4.0 \pm 0.8[3.6,3.5-4.5]$ & $3.9 \pm 0.7[3.6,3.5-3.6]$ & 0.24 \\
\hline $\begin{array}{l}\text { Exclusive breastfeeding } \\
\text { at discharge }\end{array}$ & $44(43.1)$ & $30(33.7)$ & 0.39 \\
\hline
\end{tabular}

Data are mean \pm standard deviation [median, IQR] or $\mathrm{N}(\%)$ unless otherwise specified.

Table 3: Post-surgical pain and satisfaction scores according to trial group. 


\begin{tabular}{|c|c|c|c|}
\hline Outcome & $\begin{array}{l}\text { Natural cesarean } \\
(\mathrm{N}=102)\end{array}$ & Control group $(\mathrm{N}=89)$ & $P$-value \\
\hline $\begin{array}{l}\text { VAS at the beginning of } \\
\text { surgery }\end{array}$ & $0.01 \pm 0.10[0.0,0.0-0.0]$ & $0.01 \pm 0.11[0.0,0.0-0.0]$ & 0.90 \\
\hline $\begin{array}{l}\text { VAS at the end of the } \\
\text { surgery }\end{array}$ & $0.03 \pm 0.23[0.0,0.0-0.0]$ & $0.06 \pm 0.29[0.0,0.0-0.0]$ & 0.30 \\
\hline $\begin{array}{l}\text { Need for any analgesics } \\
\text { during surgery }\end{array}$ & $6(5.9)$ & $4(4.5)$ & 0.75 \\
\hline $\begin{array}{l}\text { Need for any analgesics } \\
\text { in the immediate post } \\
\text { operation period }\end{array}$ & $43(42.2)$ & $40(45.0)$ & 0.70 \\
\hline $\begin{array}{l}\text { Maximal VAS reported } \\
\text { by each women during } \\
\text { the first } 24 \text { hours after } \\
\text { CD }\end{array}$ & $4.3 \pm 2.1[5.0,2.0-6.0]$ & $4.8 \pm 2.1[5.0,2.0-6.0]$ & 0.15 \\
\hline $\begin{array}{l}\text { Need for any sedatives } \\
\text { during surgery }\end{array}$ & $11(10.8)$ & $15(16.9)$ & 0.22 \\
\hline Maternal satisfaction & $5.4 \pm 0.74[5.6,5.1-5.9]$ & $5.4 \pm 0.5[5.6,5.3-5.8]$ & 0.68 \\
\hline
\end{tabular}

Data are mean \pm standard deviation [median, IQR] or $\mathrm{N}(\%)$ unless otherwise specified.

VAS; Visual Analog Scale from 0 to $10 \mathrm{~cm}$ where no pain scored 0 and worst pain scored 10.

Table 4: Neonatal outcomes according to trial group.

\begin{tabular}{|c|c|c|c|c|}
\hline Outcome & $\begin{array}{l}\text { Natural cesarean } \\
(\mathrm{N}=102)\end{array}$ & $\begin{array}{l}\text { Control group } \\
(\mathrm{N}=89)\end{array}$ & $P$-value & $\mathrm{RR}(95 \% \mathrm{CI})$ \\
\hline $\begin{array}{l}\text { Neonatal } \\
\text { birthweight, gr }\end{array}$ & $\begin{array}{l}3273.2 \pm 479.4 \\
{[3222.5,} \\
2975.0-3445.0]\end{array}$ & $\begin{array}{l}3285.6 \pm 481.2 \\
{[3235.0,2930.0-} \\
3525.0]\end{array}$ & 0.85 & $* * *$ \\
\hline Male neonates & $48(47.1)$ & $41(46.1)$ & 0.89 & $1.019[0.781,1.328]$ \\
\hline $\begin{array}{l}\text { Cord artery } \mathrm{pH}< \\
7.1\end{array}$ & $3(2.94)$ & $1(1.14)$ & 0.63 & $1.410[0.788,2.521]$ \\
\hline $\begin{array}{l}\text { Neonatal } \\
\text { hypothermia }{ }^{1} \text { at } \\
\text { admission to } \\
\text { nursery }\end{array}$ & $21(20.6)$ & $11(12.4)$ & 0.13 & $1.289[0.961,1.728]$ \\
\hline NICU admission & $6(5.9)$ & $1(1.1)$ & 0.12 & $0.299[0.048,1.845]$ \\
\hline $\begin{array}{l}\text { Neonatal } \\
\text { hypoglycemia }{ }^{2} \text { at } \\
\text { admission to } \\
\text { nursery }\end{array}$ & $4(3.9)$ & $3(3.4)$ & 0.99 & $0.932[0.484,1.796]$ \\
\hline Neonatal jaundice & $13(12.8)$ & $10(11.2)$ & 0.75 & $0.937[0.637,1.378]$ \\
\hline $\begin{array}{l}\text { Any neonatal } \\
\text { morbidity }{ }^{3}\end{array}$ & $37(36.3)$ & $20(22.5)$ & 0.04 & $0.747[0.577,0.968]$ \\
\hline
\end{tabular}

Data are mean \pm standard deviation [median, IQR] or $\mathrm{N}(\%)$ unless otherwise specified.

NICU; Neonatal Intensive Care Unit.

${ }^{1}$ Hypothermia; Temperature $<36^{\circ} \mathrm{C}$. 
${ }^{2}$ Hypoglycemia; glucose level [?] $40 \mathrm{mg} / \mathrm{dL}$

${ }^{3}$ Any neonatal morbidity includes one or more of the following adverse outcomes: Neonatal hypothermia, NICU admission, Neonatal hypoglycemia, and Neonatal jaundice.

\section{Legends of figures}

Figure 1. Trial profile.

Figure 1. Trial profile.

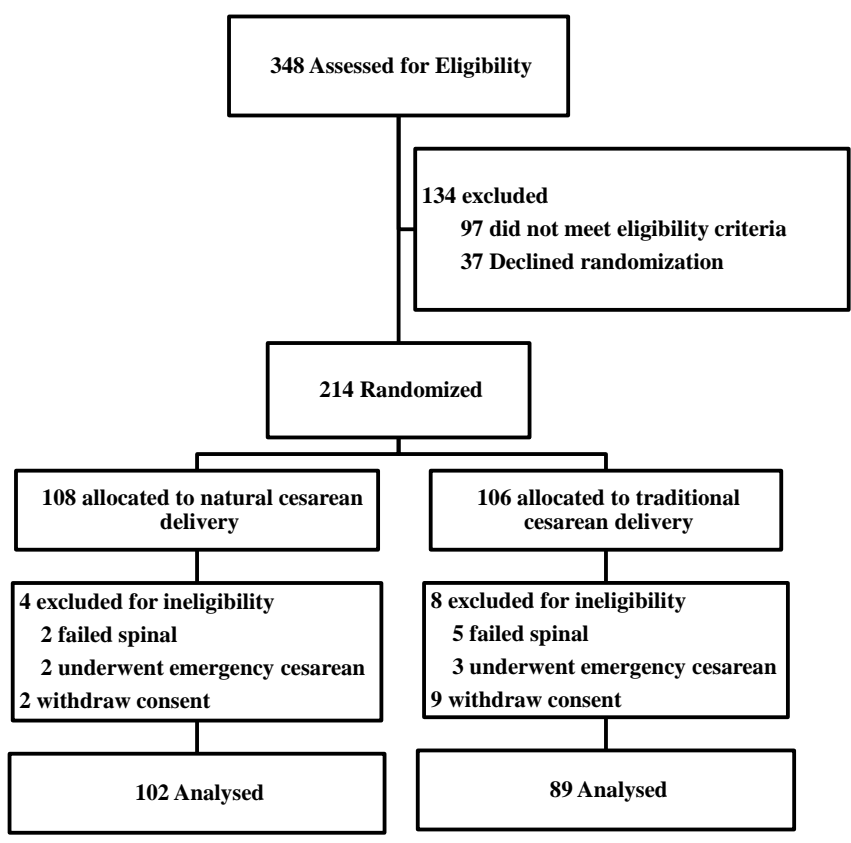

